\title{
Review of: "First principles computation of composition dependent elastic constants of omega in titanium alloys: implications on mechanical behavior"
}

Carlos Roberto Grandini, FBSE ${ }^{1}$

1 Universidade Estadual Paulista

Potential competing interests: The author(s) declared that no potential competing interests exist.

In the last decades, titanium alloys have been widely used in prostheses due to the harmlessness of the material and its low reactivity by not generating toxic products with good osseointegration. Titanium alloys are also used in air motors, aerospace structures, capacitors, valves, hip implants, fasteners, and dental casings due to its corrosion resistance characteristics and high mechanical stability. The current work investigates $\beta$-stabilizers' effect of $\beta$-stabilizers on the formation and the elastic properties of $\omega$-phase using first-principles calculations and experimental work. Different concentrations of $\beta$-stabilizers were used to analyze their effect on the $\beta \rightarrow \omega$ phase transformation. It is an exciting paper that may add very interesting knowledge for producing titanium alloys with $\beta$-stabilizers elements and good mechanical properties for several applications. 